\begin{abstract}
Revista $\quad$ ISSN 2007-6940
\section{Aplicaciones de enmiendas orgánicas y microorganismos para optimizar parámetros del suelo y rendimiento del nogal pecanero}

Applications of organic amendments and microorganisms to optimize soil parameters and performance of pecanero nogal

Piña-Ramírez F.J. ${ }^{1 凶}$, Soto-Parra J.M ${ }^{1}$, Garcia-Muñoz S.A ${ }^{1}$, Yañez-Muñoz R.M ${ }^{1}$, Perez-Leal R ${ }^{1}$.

${ }^{1}$ Facultad de Ciencias Agrotecnológicas, Universidad Autónoma de Chihuahua. Calle Escorza No. 900, C.P. 31000, Chihuahua Chih, México. Teléfono (614) 157-3705

${ }^{\bowtie}$ Autor para correspondencia: fpinar615@hotmail.com
\end{abstract}

Recibido: 11/10/2019

Aceptado: 19/11/2019

\title{
RESUMEN
}

El cultivo de nogal pecanero en el estado de Chihuahua es una actividad en crecimiento. Las zonas donde se cultiva el nogal presentan una serie de problemas, como son falta de agua, alta salinidad y alto contenido de sodio, baja nutrición en los suelos, bajo contenido de materia orgánica, entre otras, lo que provoca una disminución de la producción de nuez pecanera. El objetivo del presente estudio fue evaluar las aplicaciones al suelo de enmiendas cálcicas, orgánicas y microorganismos para los componentes del rendimiento y contenido de macronutrientes en el nogal pecanero. Los factores que se aplicaron y que influyeron en todas las variables de respuesta de los componentes de rendimiento y contenido de macronutrientes son: $\mathrm{Ca} / \mathrm{CaCO}_{3} 75.0$ y $147.4 \mathrm{~kg} \mathrm{ha}^{-1}$, Hongos Micorrízicos 20.7 y $17.2 \mathrm{~kg}$ $\mathrm{ha}^{-1}$, Humus Liquida (Hu_Liq) 750 y $1083.1 \mathrm{~L} \mathrm{ha}^{-1}$, Humus Solida (Hu_sol) 1671.2 y $1632.6 \mathrm{~kg} \mathrm{ha}^{-1}$. Las variables de respuesta que se evaluaron y fueron las más importantes para los componentes del rendimiento son: porcentaje de nuez comestible, nueces por kilogramo y peso promedio de nuez. Mientras que para el contenido de macronutrientes del suelo en nogal fueron: calcio/porcentaje de saturación de bases, calcio/(potasio+magnesio) y sodio.

Palabras clave: carbonato de calcio, micorrizas, humus solida, humus liquida y Trichoderma

\begin{abstract}
The cultivation of pecan walnut in the state of Chihuahua is a growing activity. The areas where the walnut is grown presents a series of problems which are lack of water, high salinity and high sodium content, low nutrition in the soil, low organic matter content, which causes a decrease in nut production pecanera. Therefore, the objective of this study was to evaluate the applications to the soil of calcium, organic and microorganism amendments for the components of the yield and content of macronutrients
\end{abstract}


in pecan walnut. The factors that were applied and that influenced all the response variables of the macronutrient yield and content components are: $\mathrm{Ca} / \mathrm{CaCO} 375.0$ and $147.4 \mathrm{~kg}$ ha-1, Mycorrhizal Fungi 20.7 and $17.2 \mathrm{~kg}$ ha-1, Liquid Humus (Hu_Liq ) 750 and 1083.1 L ha-1, Humus Solida (Hu_sol) 1671.2 and $1632.6 \mathrm{~kg}$ ha-1. The response variables that were evaluated and were the most important for the yield components are: percentage of edible nut, nuts per kilogram and average nut weight. While for the macronutrient content of the walnut soil were: calcium / base saturation percentage, calcium / (potassium + magnesium) and sodium.

Keywords: calcium carbonate, mycorrhizae, solid humus, liquid humus and Trichoderma

\section{INTRODUCCIÓN}

La superficie cultivada de nogal pecanero a nivel mundial es de $487 \mathrm{mil} \mathrm{ha}^{-1}$, sobresaliendo China en la producción total de nueces con el $48 \%$. En el año 2017, México participó con una superficie de $123 \mathrm{mil} \mathrm{ha}^{-1}$, con una producción de $147 \mathrm{mil} \mathrm{t}^{-1}$ con un rendimiento promedio de $1.6 \mathrm{~T} / \mathrm{ha}^{-1}$. Siendo el estado de Chihuahua, México, el mayor productor de nuez en el país, con una producción en el año 2017 de 93 mil t $^{-1}$ generando un ingreso de 7 mil 450 millones de pesos (SIAP, 2019). Las variedades más cultivadas en el estado de Chihuahua, México, son Western Schley y Wichita (Ojeda et al., 2003).

En el estado de Chihuahua el cultivo de nogal se encuentra en amplio crecimiento, pero los principales problemas del cultivo son la falta de agua, problemas nutricionales, materia orgánica baja, alta salinidad en los suelos así como altas concentraciones de sodio, dichos factores limitan la productividad y calidad de la nuez pecanera. La raíz del nogal es pivotante, no tiene pelos absorbentes, son las raicillas alimentadoras las que absorben agua y nutrimentos, la mayoría de las cuales están micorrizadas (Brison, 1976). Los hongos que han sido reportados asociados con el nogal pecanero pertenecen a los géneros Astraeus,
Gyrodon, Pisolithus, Russula, Scleroderma, Tuber y Tylopilus (Taber et al., 1982; Smith y Read, 1998).

El nogal pecanero se cultiva en las regiones semiáridas, donde los suelos son pobres en materia orgánica, fertilidad y $\mathrm{pH}$ alcalino siendo la fertilización una práctica clave para la obtención de mayor producción y calidad de fruto. De manera que un contenido alto de materia orgánica en el suelo, de $5 \%$ o más, tiene un gran efecto en el crecimiento de los árboles: a) alta disponibilidad de nutrimentos, por lo cual las deficiencias son menores o no ocurren; b) mayor retención de agua; c) mayor aireación de la raíz; y d) fuerte actividad microbiana (Wood, 2009).

Los suelos afectados por sales (sodio), pueden ser recuperados con la adición de abonos orgánicos los cuales mejoran la estructura y permeabilidad del suelo, el uso de enmiendas químicas basadas en el empleo de sales cálcicas de alta solubilidad que intercambian el sodio por calcio y la aplicación de ácidos o sustancias formadoras de éstos (Tejada et al., 2006; Aceves, 2011). Por lo que el objetivo del presente estudio fue evaluar las aplicaciones al suelo de enmiendas cálcicas, orgánicas y microorganismos para los componentes del 
rendimiento y contenido de macronutrientes en el nogal pecanero.

\section{MATERIALES Y MÉTODOS}

El estudio se realizó en la huerta el Edén, la cual se encuentra localizada en el municipio de Aldama, Chihuahua. Los árboles utilizados para dicha investigación fueron de la variedad 'Western Scheley' plantados a 12 x $12 \mathrm{~m}^{-1}$ en el año 1982. El municipio de Aldama se localiza en las coordenadas: Longitud $106^{\circ} 13^{\prime} 22.8^{\prime}$ ' $\mathrm{W}$ a 104'39'43.2' 'W, Latitud: 28 $33^{\circ} 21.96^{\prime}{ }^{\prime} \mathrm{N}$ a $29^{\circ} 40^{\prime} 17.04^{\prime \prime} \mathrm{N}$ y tiene una extensión de $9,835.90 \mathrm{~km}^{2}$, el clima es desértico, la temperatura media anual de la zona norte del municipio va de los 16 a $\operatorname{los} 18^{\circ} \mathrm{C}$, y en la zona sur entre los 18 y los $20^{\circ} \mathrm{C}$, La precipitación pluvial es escasa, ocurriendo mayormente en los meses de agosto y septiembre, el promedio anual de casi todo el territorio va de 200 a 300 mm (INEGI, 2019).

Para la generación de los tratamientos se utilizó una estructura Taguchi L16 (Minitab, 2006), con 6 factores: año, $\mathrm{CaCO} 3$, Humus Solido (Hum_Sol), Humus Liquido (Hum_Liq), Micorrizas y Trichoderma como factor dinámico; 4 niveles por cada factor resultando en 16 tratamientos (Cuadro 1), cada tratamiento con 3 repeticiones, cada repetición consistió en un árbol de nogal pecanero. Las variables de respuesta para los componentes del rendimiento son: Producción, Nueces por kilogramo, Porcentaje de nuez comestible, Peso promedio de nuez, Índice de alternancia e índice de productividad a largo plazo.

Cuadro 1. Factores y niveles estructura Taguchi L16, 3 repeticiones; factor dinámico Trichoderma

\begin{tabular}{ccccc}
\hline \multicolumn{5}{c}{ Factores / Niveles kg L ha ${ }^{-1}$} \\
\hline Concent. X & Ca_CaCO3 & Hum Lomb & Lix_Lomb & Micorrizas \\
0 & 0 & 0 & 0 & 0 \\
1 & 7.5 & 150 & 75 & 1.5 \\
10 & 75 & 1500 & 750 & 15 \\
20 & 150 & 3000 & 1500 & 30 \\
Media simple & 75 & 1500 & 750 & 15 \\
\hline
\end{tabular}

Dada la estructura factorial Taguchi L16 para la generación de tratamientos, el análisis estadístico se realizó mediante superficie de respuesta lineal y cuadrática completa, ajustando la superficie para determinar los niveles de los factores para respuesta óptima. Dicha técnica se emplea cuando cada factor tiene tres o más niveles; se estima una superficie de respuesta por regresión con el método de mínimos cuadrados; para ello se utilizó el paquete estadístico SAS (SAS Institute Inc., SAS/STAT Software: Usage and Reference, Version 6, First Edition, Cary, NC: SAS Institute Inc., 1989). El análisis para cada variable de respuesta incluyó tres etapas: 1) Análisis de la regresión y la contribución de cada factor al ajuste de la regresión; 2) análisis canónico de la superficie de respuesta para 
determinar la forma de la curva de aquellos factores que tuvieron respuesta significativa lineal, cuadrática e interacción de factores y 3 ) los valores predichos según se haya seleccionado la respuesta mínima o máxima de acuerdo con el rango original de los datos; se determina así mismo el porcentaje de incremento o decremento de la variable de respuesta y de cada uno de los factores para alcanzar el máximo o mínimo valor requerido.

Entonces, se resume el comportamiento de todas las variables de respuesta (agrupadas o no por categorías) en un cuadro donde se especifican los factores y la media simple para cada uno de ellos, se toman los eigenvalores resultantes expresados como porcentajes de la media, positivos o negativos según sea el caso; la contribución de los eigenvectores se expresa con signos redondeados a partir de 0.25 (es decir se parte del primer cuartil o mayor) tal que $0.2501 \leq+\leq 0.3749,0.3750 \leq++\leq$ $0.6249,0.6250 \leq+++\leq 0.8749,++++>$ 0.8750 , lo mismo sería para el caso de eigenvectores negativos, de esta manera se pondera cuales factores son los que más influyen en esa variable.

Se obtiene la frecuencia de signos para cada eigenvalor y el total para los eigenvalores presentes, este total se multiplican por 0.20 (se seleccionan aquellos factores cuya frecuencia de signos sea mayor al 20\% de los signos observados indistintamente positivos o negativos), posteriormente se obtiene el total de signos positivos y negativos, puesto que los eigenvectores positivos son considerablemente mayores a aquellos negativos, se seleccionarán aquellos factores de mayor peso cuya frecuencia de signos (coeficientes de eigenvectores) sea mayor al 020, para seleccionar aquellos factores de mayor peso de manera general para todas las variables de respuesta; de los factores seleccionados se obtiene la dosis máxima observada (siempre y cuando hayan sido seleccionados dentro de cada variable respuesta). Un subsecuente análisis para apoyar la discusión estará conformado por la superficie de respuesta gráfica (lineal, cuadrática e interacción que hayan resultado significativas), de esta manera se calcula si los factores son independientes, presentan sinergismo o antagonismo y con ello se obtendrá el factor o factores más críticos de manera general para el estudio.

El análisis de superficie de respuesta contempla los siguientes pasos: 1) ajuste del modelo y análisis de varianza para estimar los parámetros. a) si los tipos de efectos son lineales, cuadráticos o de productos cruzados, y cuál es la contribución de cada factor en el ajuste estadístico; 2) correlación canónica para investigar la forma de la superficie de respuesta predicha 3) Análisis de cordillera (ridge) para la búsqueda de la óptima respuesta. Los eigenvalores y eigenvectores del análisis canónico caracterizan la forma de la superficie de respuesta; los eigenvalores señalan la dirección de la principal orientación de la superficie, y los signos y magnitudes de los eigenvectores asociados proporcionan la forma de la superficie en esas direcciones. Eigenvalores positivos indican direcciones de la curvatura hacia arriba y eigenvalores negativos indican direcciones de la curvatura hacia abajo. El eigenvector para el eigenvalor más grande da la dirección de ascenso pronunciado a partir del punto fijo, si es positivo, o descenso pronunciado, si es negativo. Los eigenvectores correspondientes a eigenvalores pequeños $\mathrm{o}$ cero señalan direcciones de aplanado relativo. Para determinar si la solución es un máximo o un mínimo, se observa el signo de los 
eigenvalores: $\mathrm{Si}$ los eigenvalores son todos negativos la solución es un máximo; si son todos positivos la solución es un mínimo, si tienen signos mezclados la solución es un punto silla (saddle) y si contienen ceros la solución es un área aplanada

\section{RESULTADOS Y DISCUSIÓN}

Componentes de rendimiento y calidad de frutos.

Los factores que sobresalieron en la obtención de los componentes del rendimiento en el nogal pecanero son: hongos Micorrízicos $20.7 \mathrm{~kg} \mathrm{ha}^{-1}$, Carbonato de Calcio $\left(\mathrm{Ca} / \mathrm{CaCO}_{3}\right) 75.0 \mathrm{~kg} \mathrm{ha}^{-1}$, Humus Liquido (Hu_Liq) $750.0 \mathrm{~L} \mathrm{ha}^{-1} \mathrm{y}$ Humus Solido (Hu_Sol) $1671.2 \mathrm{~kg} \mathrm{ha}^{-1}$.

Para los componentes del rendimiento (Cuadro 2) las variables evaluadas las que sobresalieron fueron: Porcentaje de nuez comestible, Nueces por kilogramo, Peso promedio de nuez. Los factores que presentaron un mayor impacto en los componentes del rendimiento fueron: Hongos Micorrízicos $15.0 \mathrm{~kg} \mathrm{ha}^{-1}$, Carbonato de Calcio $75 \mathrm{~kg} \mathrm{ha}^{-1}$, Humus Solido (Hu_Sol) $1500.0 \mathrm{~kg} \mathrm{ha}^{-1}$ y Humus Liquido (Hu_Liq) 750 $\mathrm{kg} \mathrm{ha}^{-1}$, es necesario la aplicación de estos factores para la obtención de los valores más altos en las variables evaluadas. Trichoderma no favoreció ninguna de las variables evaluadas.

En el porcentaje de nuez comestible la media fue de $59.0 \%$, fluctuando esta variable desde
58.2 a $60.0 \%$ de almendra; la norma Mexicana NMX-FF-084-SCFI-2009 establece las especificaciones de calidad para la nuez pecanera con cascara, la cual establece que Nueces Calidad I del tipo Mejoradas deben tener un rendimiento igual o mayor al $54 \%$ de contenido comestible; los datos obtenidos en dicha investigación está por encima de los aprobados por dicha norma. Para la obtención de dichos valores es necesario la aplicación de los factores con mayor peso en el porcentaje de almendra.

En la variable Nueces por Kilogramo la media en los tres años evaluados fue de 161 nueces. Para la disminución de nueces desde 171 a 152 Nueces $\mathrm{kg}^{-1}$, es necesario aplicar $10.3 \mathrm{~kg} \mathrm{ha}^{-1} \mathrm{de}$ hongos micorrízicos, $\mathrm{Ca} / \mathrm{CaCO}_{3} 52.6 \mathrm{~kg} \mathrm{ha}^{-1}$, siendo los factores de mayor peso para dicha variable.

La norma Mexicana NMX-FF-084-SCFI-2009 clasifica la nuez como Grande cuando son de 140 a 170 nueces por kilogramo; la nuez evaluada en nuestro trabajo está dentro de dicho rango. Lagarda et al., (1998), en la Región Lagunera, estudiando la calidad de la nuez durante nueve años de evaluación, reportan 139 nueces por $\mathrm{kg}^{-1}$ para la variedad Western Schley. En nuestro estudio, dicho valor estuvo por encima de lo reportado por este investigador. A mayor numero de nueces $\mathrm{kg}^{-1}$, nos indica que las nueces son más pequeñas y por lo tanto, su calidad es menor. 
Piña-Ramírez et al., 2019

Cuadro 2. Componentes de rendimiento en nogal tratado con carbonato de calcio, materia orgánica y promotores del desarrollo vegetal.

\begin{tabular}{|c|c|c|c|c|c|c|c|c|}
\hline \multirow[b]{2}{*}{ Eigenvalores } & Año & Trichoderma & & Hu_Sol & Hu_Liq & Micorrizas & \multicolumn{2}{|c|}{ Eigenvectores } \\
\hline & 3 & 5.0 & $\begin{array}{c}/ \mathrm{CaCO}_{3} \\
\mathbf{7 5 . 0}^{\mathrm{T}}\end{array}$ & 1500.0 & 750.0 & 15.0 & Total & $\begin{array}{l}\text { Frec. + / } \\
-\end{array}$ \\
\hline \multicolumn{9}{|c|}{ Producción $\mathrm{t} \mathrm{ha}^{-1} \mu 2.11(1.28-3.14)$} \\
\hline $44.1^{\mathrm{U}}$ & $++++\mathrm{W}$ & & & & & & 4 & $4 / 0$ \\
\hline 26.6 & & & & ++ & & +++ & 5 & $5 / 0$ \\
\hline-32.3 & & & ++ & + & +++ & & 6 & $6 / 0$ \\
\hline Frecuencia & 4 & 0 & 2 & 3 & 3 & 3 & $15^{\mathrm{Y}}$ & $15 / 0$ \\
\hline $\mathrm{Kg}-\mathrm{L} \mathrm{ha}^{-1}$ & & 4.6 & 64.3 & 1331.2 & 735.9 & 16.7 & Selecc & ín $\geq 3$ \\
\hline \multicolumn{9}{|c|}{ Nueces por kilogramo $\mu 161(171-152)$} \\
\hline 6.2 & & & ++ & & +++ & ++ & 7 & $7 / 0$ \\
\hline 3.0 & & & +++ & & & --- & 6 & $3 / 3$ \\
\hline-3.99 & ++++ & & & & & & 4 & $4 / 0$ \\
\hline-9.39 & & & & ++++ & - & ++ & 7 & $6 / 1$ \\
\hline Frecuencia & 4 & 0 & 5 & 4 & 4 & 7 & 24 & $20 / 4$ \\
\hline $\mathrm{Kg}-\mathrm{L} \mathrm{ha}^{-1}$ & & 5.1 & 52.6 & 383.3 & 810.2 & 10.3 & \multicolumn{2}{|c|}{ Selección $\geq 5$} \\
\hline \multicolumn{9}{|c|}{ Porcentaje de nuez comestible $\mu 59.0(60.0-58.2 \%)$} \\
\hline 2.53 & & & ++++ & + & + & & 6 & $6 / 0$ \\
\hline 1.28 & & & & & + & ++++ & 5 & $5 / 0$ \\
\hline-0.88 & & & -- & & +++ & + & 6 & $4 / 2$ \\
\hline-1.38 & & ++++ & & & & & 4 & $4 / 0$ \\
\hline-1.56 & & & & ++++ & & + & 5 & $5 / 0$ \\
\hline Frecuencia & 0 & 4 & 6 & 5 & 5 & 6 & 26 & $24 / 2$ \\
\hline $\mathrm{Kg}-\mathrm{L} \mathrm{ha}^{-1}$ & & 5.0 & 75.0 & 1500.0 & 750.0 & 15.0 & Selecc & ión $\geq 5$ \\
\hline \multicolumn{9}{|c|}{ Peso promedio de nuez $\mu 6.27(5.85-6.79)$} \\
\hline 35.87 & & & & +++ & - & ++ & 6 & $5 / 1$ \\
\hline-11.23 & & & -- & & & +++ & 5 & $3 / 2$ \\
\hline-23.79 & & & +++ & & +++ & + & 7 & $7 / 0$ \\
\hline Frecuencia & 0 & 0 & 5 & 3 & 4 & 6 & 18 & $15 / 3$ \\
\hline $\mathrm{Kg}-\mathrm{L} \mathrm{ha}^{-1}$ & & 5.4 & 64.6 & 2612.1 & 381.7 & 20.7 & Selecc & ión $\geq 4$ \\
\hline \multicolumn{9}{|c|}{ Índice de alternancia $\mu 50.5(7.98-6.84 \%)$} \\
\hline 220.14 & & & ++++ & & + & & 5 & $5 / 0$ \\
\hline-213.39 & & & & +++ & -- & ++ & 7 & $5 / 2$ \\
\hline Frecuencia & & 0 & 4 & 3 & 3 & 2 & 12 & $10 / 2$ \\
\hline $\mathrm{Kg}-\mathrm{L} \mathrm{ha}^{-1}$ & & 5.2 & 62.6 & 1671.2 & 512.9 & 17.1 & \multicolumn{2}{|c|}{ Selección $\geq 3$} \\
\hline
\end{tabular}


Piña-Ramírez et al., 2019

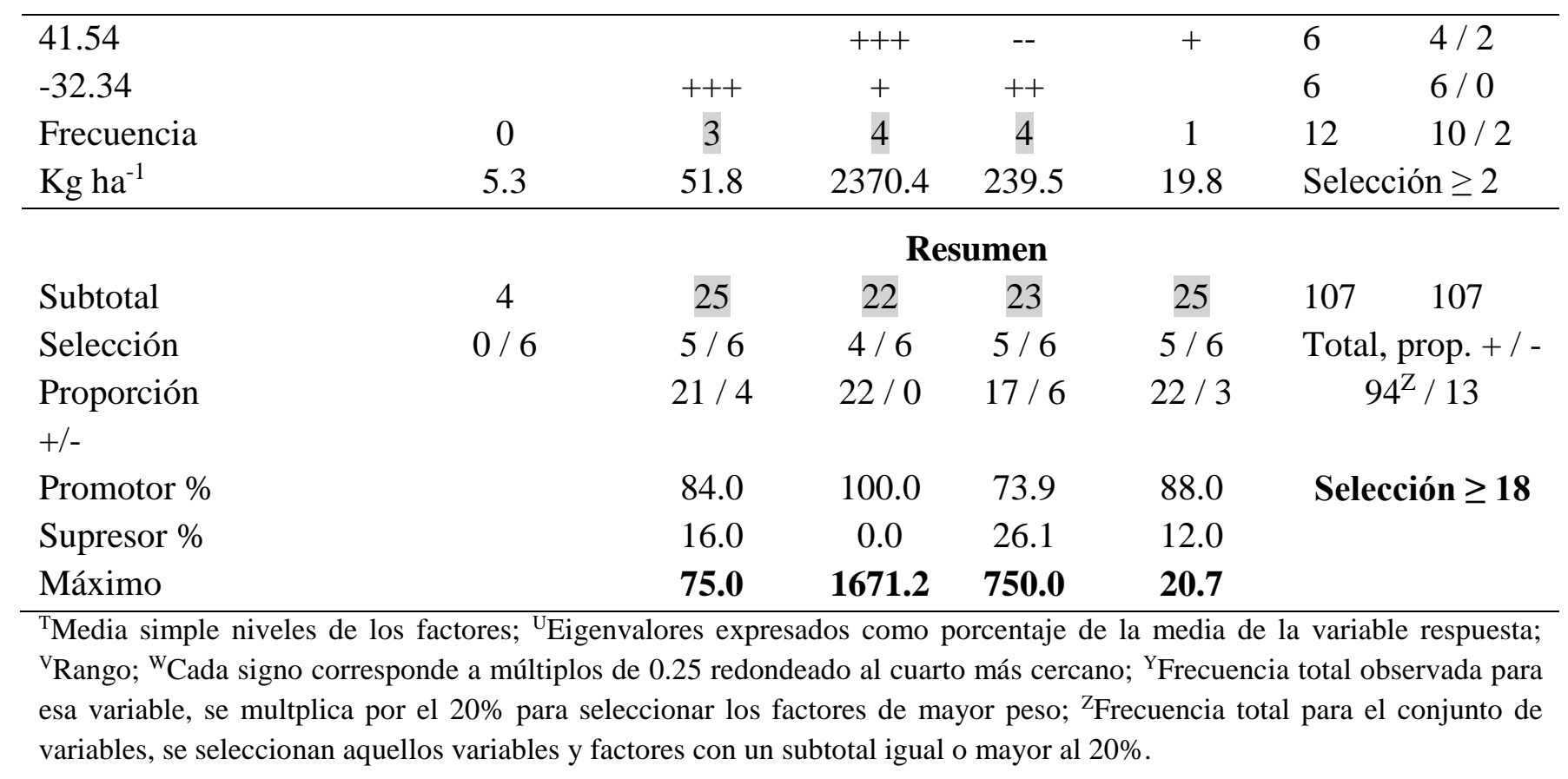

En el peso promedio de nuez la media fue de $6.27 \mathrm{gr}$, fluctuando desde 5.85 a $6.79 \mathrm{gr} / \mathrm{nuez}$. Para la obtención de nueces de mayor peso es necesario la aplicación de los siguientes factores Hongos Micorrízicos $20.7 \mathrm{~kg} \mathrm{ha}^{-1}$, $\mathrm{Ca} / \mathrm{CaCO}_{3} 64.6 \mathrm{~kg} \mathrm{ha}^{-1}$ y Hu_Liq $381.7 \mathrm{~L} \mathrm{ha}^{-1}$. Esto se debe a que el hongo trasloca a la raíz agua y nutrientes, y la raíz pasa al hongo carbohidratos $\mathrm{y}$ otras sustancias nutritivas
(Castellano y Molina, 1999; Tarango, 2004).

Contenido de macronutrientes en suelo

En el contenido de macronutrientes del suelo (Cuadro 3) las variables valoradas son contenido de Nitratos, Fósforo, Potasio, Calcio, Magnesio, Sodio, Calcio/porcentaje de saturación de bases y Calcio/ (Potasio+Magnesio).

Cuadro 3. Contenido de macronutrientes de suelo en nogal tratado con carbonato de calcio, materia orgánica y promotores del desarrollo vegetal.

\begin{tabular}{|c|c|c|c|c|c|c|}
\hline \multirow[b]{2}{*}{ Eigenvalores } & \multirow{2}{*}{$\begin{array}{c}\mathrm{Ca} / \mathrm{CaCO}_{3} \\
\mathbf{7 5 . 0}^{\mathrm{T}}\end{array}$} & \multirow{2}{*}{$\begin{array}{l}\text { Hu_Sol } \\
\mathbf{1 5 0 0 . 0}\end{array}$} & \multirow{2}{*}{$\begin{array}{c}\text { Hu_Liq } \\
\mathbf{7 5 0 . 0}\end{array}$} & \multirow{2}{*}{$\begin{array}{c}\text { Micorrizas } \\
\mathbf{1 5 . 0}\end{array}$} & \multicolumn{2}{|c|}{ Eigenvectores } \\
\hline & & & & & Total & Frec. $+/-$ \\
\hline \multicolumn{7}{|c|}{ Nitratos $\mu$ 123.1 $\left(\mathbf{1 0 7 . 9}-321.1 \mathrm{~kg} \mathrm{ha}^{-1}\right)$} \\
\hline $228.7^{\mathrm{U}}$ & $++++{ }^{\mathrm{W}}$ & & + & & 5 & $5 / 0$ \\
\hline-218.0 & & ++ & - & ++ & 5 & $4 / 1$ \\
\hline Frecuencia & 4 & 2 & 2 & 2 & $10^{\mathrm{Y}}$ & $9 / 1$ \\
\hline $\mathrm{Kg}-\mathrm{L} \mathrm{ha}^{-1}$ & 105.1 & 1632.6 & 963.1 & 14.9 & Selección $\geq 2$ & \\
\hline \multicolumn{7}{|c|}{ Fósforo $\mu 19.8\left(3.6-35.3 \mathrm{mg} \mathrm{kg}^{-1}\right)$} \\
\hline 291.6 & ++++ & & & & 4 & $4 / 0$ \\
\hline 40.5 & & & +++ & ++ & 5 & $5 / 0$ \\
\hline-158.49 & & +++ & & ++ & 5 & $5 / 0$ \\
\hline
\end{tabular}


Piña-Ramírez et al., 2019

\begin{tabular}{|c|c|c|c|c|c|c|}
\hline Frecuencia & 4 & 3 & 3 & 4 & 14 & $14 / 0$ \\
\hline $\mathrm{Kg}-\mathrm{L} \mathrm{ha}^{-1}$ & 147.4 & 1125.6 & 777.5 & 15.9 & \multicolumn{2}{|c|}{ Selección $\geq 3$} \\
\hline \multicolumn{7}{|c|}{ Potasio $\mu 386.7\left(139.5-605.9 \mathrm{mg} \mathrm{kg}^{-1}\right)$} \\
\hline 126.3 & ++++ & & & & 4 & $4 / 0$ \\
\hline 52.0 & & & +++ & ++ & 5 & $5 / 0$ \\
\hline-63.6 & & +++ & - & ++ & 6 & $5 / 1$ \\
\hline Frecuencia & 4 & 3 & 4 & 4 & 15 & $14 / 1$ \\
\hline $\mathrm{Kg}-\mathrm{L} \mathrm{ha}^{-1}$ & 129.2 & 1511.2 & 982.4 & 17.2 & \multicolumn{2}{|c|}{ Selección $\geq 3$} \\
\hline \multicolumn{7}{|c|}{ Calcio $\mu 3276.6\left(1519.0-\underline{\left.6097.4 \mathrm{mg} \mathrm{kg}^{-1}\right)}\right.$} \\
\hline 300.4 & ++++ & & & & 4 & $4 / 0$ \\
\hline 176.9 & & & +++ & ++ & 5 & $5 / 0$ \\
\hline-226.8 & & ++ & - & ++ & 5 & $4 / 1$ \\
\hline Frecuencia & 4 & 2 & 4 & 4 & 14 & $13 / 1$ \\
\hline $\mathrm{Kg}-\mathrm{L} \mathrm{ha}^{-1}$ & 112.5 & 1503.7 & 993.1 & 16.1 & \multicolumn{2}{|c|}{ Selección $\geq 3$} \\
\hline \multicolumn{7}{|c|}{ Magnesio $\mu \underline{779.7}\left(\underline{730.3-533.6} \mathrm{mg} \mathrm{kg}^{-1}\right)$} \\
\hline 24.8 & ++++ & & & & 4 & $4 / 0$ \\
\hline 9.0 & & & +++ & ++ & 5 & $5 / 0$ \\
\hline-14.0 & & +++ & & ++ & 5 & $5 / 0$ \\
\hline Frecuencia & 4 & 3 & 3 & 4 & 14 & $14 / 0$ \\
\hline $\mathrm{Kg}-\mathrm{L} \mathrm{ha}^{-1}$ & 66.5 & 275.2 & 714.0 & 6.5 & \multicolumn{2}{|c|}{ Selección $\geq 3$} \\
\hline \multicolumn{7}{|c|}{ Sodio $\mu$ 534.4 (581.9 - 463.9) } \\
\hline 19.8 & ++++ & & & & 4 & $4 / 0$ \\
\hline-7.3 & - & + & +++ & -- & 7 & $4 / 3$ \\
\hline-13.3 & & ++++ & - & & 5 & $4 / 1$ \\
\hline Frecuencia & 5 & 5 & 4 & 2 & 16 & $12 / 4$ \\
\hline $\mathrm{Kg}-\mathrm{L} \mathrm{ha}^{-1}$ & 66.8 & 172.8 & 621.7 & 8.7 & \multicolumn{2}{|c|}{ Selección $\geq 3$} \\
\hline
\end{tabular}

\begin{tabular}{|c|c|c|c|c|c|c|}
\hline \multicolumn{7}{|c|}{ Calcio / porcentaje de saturación de bases $\mu$ 63.5 $(\mathbf{5 5 . 6}-\underline{87.0})$} \\
\hline 105.8 & ++++ & & + & & 5 & $5 / 0$ \\
\hline 61.2 & - & & +++ & ++ & 6 & $5 / 1$ \\
\hline-93.1 & & +++ & - & ++ & 6 & $5 / 1$ \\
\hline Frecuencia & 5 & 3 & 5 & 4 & 17 & $15 / 2$ \\
\hline $\mathrm{Kg}-\mathrm{L} \mathrm{ha}^{-1}$ & 104.5 & 1548.1 & 978.7 & 15.6 & \multicolumn{2}{|c|}{ Selección $\geq 3$} \\
\hline \multicolumn{7}{|c|}{$\mathrm{Ca} /($ potasio + magnesio $) \mu 2.72\left(1.42-8.01 \mathrm{meq} 100 \mathrm{~g}^{-1}\right.$ suelo $)$} \\
\hline 225.3 & ++++ & & + & & 5 & $5 / 0$ \\
\hline 146.8 & - & & +++ & ++ & 6 & $5 / 1$ \\
\hline-199.2 & & +++ & & ++ & 5 & $5 / 0$ \\
\hline Frecuencia & 5 & 3 & 4 & 4 & 16 & $15 / 1$ \\
\hline $\mathrm{Kg}-\mathrm{L} h \mathrm{~h}^{-1}$ & 124.5 & 1532.7 & 1083.1 & 16.3 & \multicolumn{2}{|c|}{ Selección $\geq 3$} \\
\hline \multicolumn{7}{|c|}{ Resumen } \\
\hline Subtotal & 35 & 24 & 29 & 28 & 116 & 116 \\
\hline Selección & $8 / 8$ & $7 / 8$ & $8 / 8$ & $7 / 8$ & \multicolumn{2}{|c|}{ Total, proporción $+/$ - } \\
\hline
\end{tabular}


Piña-Ramírez et al., 2019

\begin{tabular}{lccccc}
\hline Proporción +/- & $32 / 3$ & $24 / 0$ & $24 / 5$ & $26 / 2$ & $106^{\mathrm{Z}} / 10$ \\
Promotor \% & 91.4 & 100.0 & 82.8 & 92.9 & Selección $\geq \mathbf{2 1}$ \\
Regresor \% & 8.6 & 0.0 & 17.2 & 7.1 & \\
Máximo & $\mathbf{1 4 7 . 4}$ & $\mathbf{1 6 3 2 . 6}$ & $\mathbf{1 0 8 3 . 1}$ & $\mathbf{1 7 . 2}$ & \\
\hline
\end{tabular}

${ }^{\mathrm{T}}$ Media simple niveles de los factores; ${ }^{\mathrm{E}}$ Eigenvalores expresados como porcentaje de la media de la variable respuesta; ${ }^{\mathrm{v}}$ Rango entre paréntesis corresponde a los valores predichos a partir de la media simple, en negrillas cae por debajo del rango óptimo, subrayado por encima, de otra manera se consideran valores óptimos (Soto et al., 2008); ${ }^{\mathrm{C}} \mathrm{Cada}$ signo corresponde a múltiplos de 0.25 redondeado al cuarto más cercano; ${ }^{Y}$ Frecuencia total observada para esa variable, se multplica por el $20 \%$ para seleccionar los factores de mayor peso; ${ }^{Z}$ Frecuencia total para el conjunto de variables, se seleccionan aquellos factores con un subtotal igual o mayor al $20 \%$.

Los factores de mayor impacto sobre dichas variables fueron $\mathrm{Ca} / \mathrm{CaCO}_{3}$ en un $35 \%$, Hu_Liq en un $29 \%$, Hongos Micorrízicos en un $28 \%$ y Hu_Sol $24 \%$. Siendo el carbonato de calcio el que tuvo mayor incidencia en todas las variables de respuesta. Mientras que de las variables evaluadas las que fueron de mayor peso en el contenido de macronutrientes del suelo son: Calcio/porcentaje de saturación de bases, $\mathrm{Ca} /($ potasio+magnesio) y sodio.

En la relación de la variable $\mathrm{Ca} /$ porcentaje de saturación de bases la media fue de $63.5 \%$, dicha variable oscilo desde 55.6 hasta $87.0 \%$, lo que nos indica que la media $(63.5 \%)$ y el primer valor $(55.6 \%)$ se encuentran por abajo del optimo, mientras que el segundo valor (87.0 $\%$ ) está por encima del valor óptimo (65 a 75 $\%)$ propuesto por Graham, (1959).

La relación del $\mathrm{Ca} /($ Potasio+Magnesio) la media fue de 2.72 meq $100 \mathrm{~g}^{-1}$ suelo, fluctuando desde 1.42 a 8.01 meq $100 \mathrm{~g}^{-1}$ suelo, para obtener la fluctuación más alta es necesario la aplicación de $124.5 \mathrm{~kg} \mathrm{ha}^{-1}$ de $\mathrm{Ca} / \mathrm{CaCO}_{3}, 1083.11 \mathrm{ha}^{-1}, 16.3 \mathrm{~kg}$ ha de hongos micorrizicos y $1532.7 \mathrm{~kg} \mathrm{ha}^{-1}$ Hu_Sol. Los abonos orgánicos pueden satisfacer la demanda de nutrientes de los cultivos, reduciendo significativamente el uso de fertilizantes químicos y mejorando las características de los vegetales con-sumidos (Rodríguezet al. 2009) además, los abonos orgánicos mejoran las características de suelos que han sido deteriorados por el uso excesivo de agroquímicos y su sobre-explotación (Nieto et al. 2002). El calcio se aplica al suelo en algunas huertas nogaleras con la finalidad de contrarrestar efectos dañinos del sodio. La fuente del calcio puede provenir de la calcita $\left(\mathrm{CaCO}_{3}\right)$.

En la variable Sodio la media fue de $534.4 \mathrm{mg}$ $\mathrm{kg}^{-1}$, dicha variable fluctuó desde 581.9 hasta $463.9 \mathrm{mg} \mathrm{kg} \mathrm{kg}^{-1}$, con la aplicación de los siguientes factores $\mathrm{Ca} / \mathrm{CaCO}_{3} 66.8 \mathrm{~kg} \mathrm{ha} \mathrm{ha}^{-1}$, $\mathrm{Hu} \_S o l 172.8 \mathrm{~kg} \mathrm{ha}^{-1} \mathrm{y} \mathrm{Hu} \_$Liq $621.7 \mathrm{~L} \mathrm{ha}^{-1}$.

\section{CONCLUSIONES}

Los factores que más influyeron en los componentes de rendimiento del nogal pecanero fueron: micorrizas, carbonato de calcio, humus líquido y humus solido. Las variables de respuesta que influyeron para los componentes del rendimiento en el nogal pecanero son: porcentaje de nuez comestible, nueces por quilogramo y peso promedio de nuez.

Mientras que para el contenido de macronutrientes del suelo los factores que más

Revista Científica Biológico Agropecuaria Tuxpan 7 (2) 
Piña-Ramírez et al., 2019

influencia tuvieron fueron: carbonato de calcio, humus líquido, micorrizas y humus solido.

\section{LITERATURA CITADA}

Brison, F.R. 1976. Cultivo del nogal pecanero (tr. Federico Garza F.). México. Conafrut. 349 p.

Castellano, M. A. and R. Molina. 1999. Mycorrhizae. In:T.D. Landis, R.W. Tinus, S.E. McDonald and J.P. Barnett (eds.). The container tree nursery manual. Vol. 5. Agric. Handbook 674. USDA-FS.P. 101-167.

Davies, F.T.; V. Olalde-Portugal, M.J. Alvarado, H.M. Escamilla, R.FerreraCerrato and J.I. Espinosa. 2000. Alleviatingphosphorus stress of chile ancho pepper (Capsicum annuumL. San Luis by arbuscular mycorrhizal inoculation. J. Hortic.Sci. Biotechnol. 75:655-661.https://doi.org/10.1080/1462 0316.2000 .11511303

Finlay, R. D. 2008. "Ecological aspects of mycorrhizal simbiosis: with special emphasis on the functional diversity of interactions involving the extraradical mycelium.” J Exp Bot 59: 1115-26.

https://doi.org/10.1093/jxb/ern059

Graham, E.R. 1959. An explanation of theory and methods of soil testing. Mo. Agric. Exp. Stn. Bull. 734.

Hanna, J. D. 1987. Pecan rootstocks. In: Rom, R.C. and Carlson,R. F. (eds.). Rootstocks for fruit crops. John Wiley and Sons.USA. p. 401-410.

Instituto Nacional de Estadística y Geografía. «Chihuahua. Mapa con Regiones Hidrológicas». Archivado desde el original el 10 de marzo de 2010. Consultado el 2019. https://www.inegi.org.mx/contenidos/ap p/mexicocifras/datos_geograficos/08/08 002.pdf

Nieto G A, Murillo AB, Troyo DE, Larrinaga MJ, JL García H (2002) El uso de compostas como alternativaecológica para la producción sostenible del chile (Capsicum annum L.) en zonas áridas. Interciencia27(8): 417-421.

Ojeda, D., Reyes, A., Ramírez, H., Lagarda, A., Chavez, F.I., Uvalle, J.X., Ramírez, R.M y Romero, L. 2003. Uso eficiente de la fertilización nitrogenada en el cultivo del nogal pecanero (Carya illinoensis (Wangenh) K. Koch) Variedad Western Schley. Segunda Edición. Editorial Placido Cuadros. Granada, España. 26-50 p

Rodríguez DN, Cano RP, Figueroa VU, Favela CE, Moreno RA, Márquez HC, Ochoa ME, Preciado R (2009). Uso de abonos orgánicos en la producción de tomate en invernadero. Terra Latinoamericana 27: 319-327.

SAS. 1989. SAS Institute Inc., SAS/STAT ${ }^{\mathrm{R}}$ User's Guide, Version 6, Fourth Edition, Volume 2, Cary, NC: SAS Institute Inc. p1457-1478.

Servicio de Información Agroalimentaria y Pesca (SIAP). 2018. Atlas Agroalimentario 2018. México D.F: SIAP; 2018. 118-119 p. Disponible en: http://

https://nube.siap.gob.mx/gobmx_public aciones_siap/pag/2018/AtlasAgroalimentario-2018.

Taber, R.A., J.W. Worthington; J.M. Trappe and W.A. Taber. 1982. Mycorrhyzal 
fungi associated with native and improved varieties of pecan in Texas (Abstract). Phytopathology 72:951.

Tarango, R. S. H. 2004. Micorrizas en nogal pecanero ypistachero. Folleto Técnico No. 16 del INIFAP-Delicias. Centrode investigaciones regionales norte-centro campoexperimental Delicias, Agosto del 2004. Chihuahua, México.39 p.

Tejada, M., C. García, J. L. González, and M. T. Hernández. 2006. Use of organic amendment as a strategy for saline soil remediation: Influence on the physical, chemical and biological properties of soil. Soil Biol. Biochem. 38: 1413-1421

https://doi.org/10.1016/j.soilbio.2005.10.017
Vargas H. M., Zarate D. L. G. P., Burguete H., F. 1991. Factoriales fraccionados y superficie de respuesta, uso de paquetes estadísticos para microcomputadoras. Monografías y Manuales de Estadística y Cómputo. Vol.10 No. 1. p79-88.

Wood, B.W. 2009. Some guiding principles for successful nutrient management of orchards. En: $6^{\circ}$. Congreso Internacional de nogalero a nogalero. Coahuila, México.

Copyright (c) 2019 Piña-Ramirez F.J., S oto-Parra J.M., Garcia-Muñoz S.A, Y añez-Muñoz R.M., Perez-L eal R.

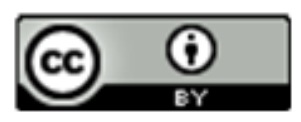

Este texto está protegido por una licencia licencia CreativeCommons 4.0.

Usted es libre para Compartir —copiar y redistribuir el material en cualquier medio o formato- y Adaptar el documento —remezclar, transformar y crear a partir del material- para cualquier propósito, incluso para fines comerciales, siempre que cumpla la condición de:

Atribución: Usted debe dar crédito a la obra original de manera adecuada, proporcionar un enlace a la licencia, e in dicar si se han realizado cambios. Puede hacerlo en cualquier forma razonable, pero no de forma tal que sugiera que tiene el apoyo del licenciante o 10 recibe por el uso que hace de la obra.

Resumendelicencia - Textocompletodelalicencia 